\title{
Bounds on Stringy Quantum Gravity from Low Energy Existing Data
}

\author{
Daniel Sudarsky \\ Center for Gravitational Physics and Geometry, Department of Physics, \\ Penn State University, University park, PA 16802, USA* \\ Luis Urrutia \\ Instituto de Ciencias Nucleares, Universidad Nacional Autónoma de México, \\ A. Postal 70-543, México D.F. 04510, México \\ Héctor Vucetich \\ Instituto Física, Nacional Autónoma de México. \\ A. Postal 70-543, México D.F. 04510, Méxicd
}

\begin{abstract}
We show that existing low energy experiments, searching for the breaking of local Lorentz invariance, set bounds upon string theory inspired quantum gravity models that induce corrections to the propagation of fields. Using the standard Observer Lorentz transformations in the D-particle recoil model we find $M \geq 1.2 \times 10^{5} M_{P}$ and $v \leq 2 \times 10^{-27} c$ for the mass and recoil speed of the Dparticle, respectively. These bounds are $\sim 10^{8}$ times stronger than the latest astrophysical bounds. These results indicate that the stringy scenario for modified dispersion relations is as vulnerable to these types of tests as the loop quantum gravity schemes.

PACS numbers: 04.60.-m, 11.25.Pm, 04.80.-y, 11.30.Cp
\end{abstract}

\footnotetext{
${ }^{*}$ On leave of absence from Instituto de Ciencias Nucleares, Universidad Nacional Autónoma de México, A. Postal 70-543, México D.F. 04510, México

${ }^{\dagger}$ On leave of absence from Observatorio Astronómico, Universidad Nacional de La Plata, La Plata, Argentina
} 


\section{INTRODUCTION}

We have recently analyzed the bounds 11 that can be extracted form direct searches of violations of Lorentz invariance [2, 3, 4, 5, 6, 7, 8, 9] that seem inevitable in the scenarios that are used to predict changes in the dispersion relations of high energy particles due to quantum gravitational effects [10, 11, 12]. Our original analysis dealt specifically with the Loop Quantum Gravity scenarios [13, 14, 15, 16, 17], however as suggested in Ref. [1], we expected similar results to apply to any such scheme and in particular to the String Theory inspired models [18, 19, 20, 21, 22, 23, 24].

The main point is that when a theory predicts that photons propagate with an energydependent velocity $v(E)$ rather than with the universal speed of light $c$, then such theory is automatically implying a breakdown of Lorentz invariance, either at the fundamental or at the spontaneous level, since such statement can be valid at best in one specific inertial frame. This selects a preferred frame of reference, where the particular form of the corrected equations of motion are valid, and one should then be able to detect the laboratory velocity with respect to that frame. Physics present us with a rather unique canonical choice for that "preferred inertial frame": that one where the Cosmic Microwave Background (CMB) looks isotropic. Our velocity $\mathbf{w}$ with respect to that frame has already been determined to be $w / c \approx 1.23 \times 10^{-3}$ by the measurement of the dipole term in the CMB by COBE, for instance 25]. Thus, it follows that the quantum gravity corrections to the corresponding particle field theory (photons, fermions and others) should contain w-dependent terms when described in our laboratory reference frame. These would lead to a breakdown of isotropy which would show up in high precision tests of rotational symmetry, that have been carried out using atomic and nuclear systems such as those described in 26, 27, 28, 29, 30, 31, 32, 33, 34].

The method of analysis is the same used in our previous work and can be thought to correspond to the application of the general framework described in the works of Ref. [6, 7], to the specific scenario in question.

The specific String Theory inspired scenario that has been considered as leading to a quantum gravity induced change in the dispersion relation of high energy particles is the so called Liouville approach to Non-critical String Theory. In this scheme our universe is identified with a 4 dimensional D-brane, which will naturally contains a type of topological defect called a D-particle. These will interact with ordinary particles, represented by strings 
in the corresponding mode, by elastic scattering, which would produce a recoil of the Dparticle. This recoil will induce a local disturbance of the space-time geometry which will in turn affect the propagation of the particle [18, 19, 20, 21, 22, 23, 24]. In the following, we shall use "brane" and "D-particle" as synonymous terms.

We take this part of the analysis directly from Ref. [24], starting with their modified Dirac equation

$$
\left[\gamma^{\mu}\left(i \partial_{\mu}-e A_{\mu}\right)-v^{i} \gamma^{0}\left(i \partial_{i}-e A_{i}\right)-m\right] \Psi=0
$$

Here $\gamma^{\mu}$ are the standard flat-space gamma matrices and $v^{i}$ is the D-particle recoil velocity in the CMB frame, where it is initially at rest. The above modified Dirac equation preserves gauge invariance due to the standard minimal coupling. In the following we set $A_{\mu}=0$. We will be concerned with a perturbative expansion in the small parameter $v^{i}$. Note that such modification of Dirac's equation, having a privileged constant vector $v^{i}$, might arise in different types of quantum gravity models.

Already at this level one might consider two alternatives in addressing the model based in Eq.(11):

(i) the first one, proposed in Ref. [24], argues that Eq.(11) is form invariant, while maintaining the meaning of $v^{i}$ as the brane recoil velocity produced by the particle-brane collision in all references frames. This property, suggested by the underlying string theory basis of (11), is realized by demanding that $v^{i}$ is embedded in a tetrad $e_{a}{ }^{\mu}$ with components;

$$
e_{0}^{0}=1, \quad e_{0}^{i}=v^{i}, \quad e_{i}^{0}=\mathbf{0}, \quad e_{i}^{j}=\delta_{i}^{j},
$$

in each reference frame. The above leads to a modified Minkowski metric $G_{\mu \nu}$ producing the following invariant in momentum space

$$
p^{\mu} G_{\mu \nu} p^{\nu}=E^{2}-2 E \mathbf{v} \cdot \mathbf{p}-\mathbf{p}^{2}, \quad p^{\mu}=(E, \mathbf{p}), \quad|\mathbf{v}| \approx \frac{|\mathbf{p}|}{M} .
$$

where $M$ is the D-particle's mass.

As stated in Ref. [23], the set of transformations leaving invariant either (2) or (3) is a subgroup of the standard Lorentz group. Application of this idea then would mean, at least, to abandon the use of the standard Lorentz transformations to connect inertial reference frames. Since one is violating Lorentz covariance, this is a possible alternative which remains to be explored in full detail. However, we must point out that it is thus 
unclear what would be the transformation properties of the Dirac wave function $\Psi$ and of the equation (11), and in which frame should we consider such description to be valid.

(ii) Here we take a more conservative approach by maintaining the full Lorentz covariance relating any two inertial frames (Observer Lorentz covariance), according to the standard model extension of Kostelecký et. al 2, 3, 4, 5, 6, 17, 8, 9]. Then we expect the metric (3) to transform as a second-rank tensor, while the brane recoil velocity is embedded in the four vector $V^{\mu}$ defined as a momentum transfer. To order $1 / M$ we have $V^{\mu}=(0, \mathbf{v})$ in the CMB frame. As expected, the collision calculation of the brane recoil velocity in each reference frame is consistent with the standard Lorentz transformation properties of the four-vector $V^{\mu}$. Also we take a perturbative approach where the brane recoil velocity (which is of order $|\mathbf{p}| / M)$ is calculated using the uncorrected energy-momentum dispersion relations. We have checked that the use of the modified dispersion relations arising from Eq.(3) does not change the results to that order.

The paper is organized as follows: In section [I we sketch the calculation of the brane recoil velocity according to the prescriptions of Ref. [18, 19, 20, 21, 22, 23]. Then (Section III) we analyze the modified Dirac equation according to the viewpoint (ii) above. Finally (Section IV) we consider some relevant experimental results which are compared with the theory's expectations and then state our conclusions.

\section{BRANE RECOIL VELOCITY}

In order to have a quantitative description of the collision process which satisfies the requirements of the theory in Ref [24], which are (in the frame where the brane is initially at rest)

- brane recoil velocity parallel to the momentum $\mathbf{p}$ of the incoming particle.

- Magnitude of the recoil velocity of order $|\mathbf{p}| / M$,

we consider a 1-dimensional description of the collision where the modeled as a large reflecting mirror of mass $M$ where the incoming particle is perpendicularly reflected.

The brane recoil velocity $\mathbf{V}$ is defined by

$$
\mathbf{V} \equiv \frac{1}{M}(\mathbf{p}-\mathbf{r})
$$


which we covariantize as

$$
V^{\mu}=\frac{1}{M}\left(p^{\mu}-r^{\mu}\right)=\left(V^{0}, \mathbf{V}\right),
$$

where $p^{\mu}, r^{\mu}$ are the standard four momenta of the in and out ordinary particles, respectively. We write $p^{\mu}=(E, \mathbf{p})$ use the standard dispersion relation $E=\sqrt{p^{2}+m^{2}}(c=1)$ together with the notation:

$$
\begin{aligned}
\mathbf{p}=p \hat{\mathbf{k}}, \quad \mathbf{r}=r \hat{\mathbf{k}}, \\
\mathbf{w}=w \hat{\mathbf{k}}, \quad \mathbf{u}=u \hat{\mathbf{k}},
\end{aligned}
$$

for the incoming and outcoming particle's momenta and D-brane velocities respectively. Using energy-momentum conservation in the non-relativistic limit, we obtain

$$
V=2 \frac{p-m w}{M+m}=V(w), \quad \mathbf{V}=V \hat{\mathbf{k}}
$$

which reduces to

$$
V=\frac{2}{M}(p-m w)+O\left(M^{-2}\right)
$$

in the large D-particle mass limit.

In the same way, the zeroth component of the recoil velocity (to order $1 / M$ ) is just

$$
V^{0}=\frac{2 w}{M}(p-m w)=w V .
$$

The above equation is the relativistic statement that

$$
W^{\mu} V_{\mu}=0
$$

which follows from the fact that $V^{\mu}$ is just $\delta W^{\mu}$ while $W^{\mu} W_{\mu}$ is constant (i.e.=1). Thus to order $O(1 / M)$ we have

$$
V_{L A B}=\gamma_{w} V_{C M B} .
$$

Coming back to vector notation, the above relations take the form

$$
\mathbf{V}=\frac{2}{M}(\mathbf{p}-m \mathbf{w}), V^{0}=\mathbf{w} \cdot \mathbf{V} .
$$

It is easily seen that $\mathbf{V}$ is Galilean invariant to first order in $w$, and is thus equal to $\mathbf{V}_{C M B}$ (to such order), so we introduce the notation

$$
\mathbf{v}=\mathbf{V}_{C M B}=\frac{2}{M} \mathbf{p}_{C M B}=\frac{2}{M}\left(\mathbf{p}_{L A B}-m \mathbf{w}\right)=\mathbf{V}_{L A B}
$$

for the Galilean-invariant D-particle recoil velocity. 
However, $V^{0}$ is not Galilean invariant

$$
V_{L A B}^{0}=\mathbf{w} \cdot \mathbf{v} \neq V_{C M B}^{0}=0
$$

and it is invariant only under the subset of Galilean transformations involving relative velocities orthogonal to the D-particle recoil velocity. But nothing in the actual laboratory experiments we will consider is restrictive in this way.

Equation (13]) is a particular case of the condition considered in Ref [23] as defining "admissible" Lorentz transformations: they should keep the magnitude of the recoil velocity invariant: $\left|\mathbf{V}_{C M B}\right|=\left|\mathbf{V}_{L A B}\right|$. The simple Galileo transformation leaves both magnitude and direction of the vector invariant.

\section{REDUCTION OF THE MODIFIED DIRAC EQUATION}

We assume that equation (11) describes the dynamics in the CMB frame. In order to obtain the corresponding description in the laboratory frame we rewrite (11) in a covariant way by introducing the laboratory $\mathrm{CMB} 4$-velocity as $W^{\mu}=\gamma(1, \mathbf{w} / c)$. Here we are imposing covariance under observer Lorentz transformations, while we are violating particle Lorentz invariance, in the notation of Ref. [7].

Let us consider the correction term

$$
\Delta D=-\gamma^{0} i v^{k} \partial_{k} \Psi
$$

The covariant extensions are

$$
\begin{aligned}
\gamma^{0} & \longrightarrow \gamma^{\mu} W_{\mu}, \\
v^{k} \partial_{k} & \longrightarrow V^{\mu}\left(g_{\mu \nu}-W_{\mu} W_{\nu}\right) \partial^{\nu},
\end{aligned}
$$

which produce

$$
\Delta D=-\left(\gamma^{\rho} W_{\rho}\right) V^{\mu}\left(g_{\mu \nu}-W_{\mu} W_{\nu}\right) \partial^{\nu} \Psi
$$

This amounts to apply an standard Lorentz boost from the CMB frame to the LAB frame.

Here $V^{\mu}$ is the recoil four velocity of the D-particle with respect to the laboratory frame. The resulting Dirac equation is then

$$
\left\{\gamma^{\mu} i \partial_{\mu}-\gamma^{\rho}\left[W_{\rho} V^{\mu}\left(\delta_{\mu}^{\nu}-W_{\mu} W^{\nu}\right)\right] i \partial_{\nu}-m\right\} \Psi=0
$$


From (10) the above equation simplifies to

$$
\left\{\gamma^{\mu} i \partial_{\mu}-\gamma^{\rho}\left[W_{\rho} V^{\nu}\right] i \partial_{\nu}-m\right\} \Psi=0
$$

which can be put in the standard form of [35, 36]. The identification of the corresponding Kostelecký parameters leads to

$$
a_{\mu}=b_{\mu}=0=H_{\mu \nu}, \quad g_{\lambda \mu \nu}=0=d_{\mu \nu}, \quad c_{\mu \nu}=-W_{\mu} V_{\nu}
$$

Let us note that $c_{\mu \nu}$ is not symmetrical in this case. Note also that we have $c_{\mu \nu} \sim W_{\mu} V^{\rho} C_{\rho \nu}$, where $C_{\rho \nu}$ is the analogous factor introduced in Ref. 11]. This makes explicit the difference between the theories considered there and in Ref. [24].

According to [35, 36] the corresponding Hamiltonian in the non-relativistic limit is

$$
h=m+\frac{p^{2}}{2 m}-m c_{00}+m\left(c_{0 j}+c_{j 0}\right) \frac{p_{j}}{m}-m\left(c_{j k}+\frac{1}{2} c_{00} \delta_{j k}\right) \frac{p_{j} p_{k}}{m^{2}}
$$

up to fourth order in the momenta. Here $p_{j}$ are the components of $-\mathbf{p}$. Using (201) we find

$$
\begin{gathered}
c_{00}=-\mathbf{w} \cdot \mathbf{v}, \quad c_{0 i}=v^{i}\left(1+\frac{w^{2}}{2}\right), \\
c_{i 0}=(\mathbf{v} \cdot \mathbf{w}) w^{i}, \quad c_{i j}=-w^{i} v^{j},
\end{gathered}
$$

in the non-relativistic approximation, where we have kept terms up to second order in $\mathbf{w}$. In this way, (21) reduces to

$$
h=m(1+\mathbf{w} \cdot \mathbf{v})-\left(1+\frac{w^{2}}{2}\right) \mathbf{p} \cdot \mathbf{v}+(1+\mathbf{w} \cdot \mathbf{v}) \frac{p^{2}}{2 m}+\frac{1}{m}[\mathbf{v} \cdot(\mathbf{p}-m \mathbf{w})](\mathbf{w} \cdot \mathbf{p})
$$

Let us emphasize that the above Hamiltonian describes the non-relativistic, linear in $\mathbf{v}$ (the D-particle's recoil velocity in the CMB frame) corrections to the spin 1/2 particle dynamics in the laboratory frame. As it will be apparent in the sequel, the term $-\mathbf{p} \cdot \mathbf{v}$ effectively contributes to the term $\frac{p^{2}}{2 m}$, which is not relevant for clock comparison experiments.

Substituting (13) in Eq. (23) the final Hamiltonian is

$$
h=m\left(1-\frac{2 m}{M} \mathbf{w}^{2}\right)+\left(1-\frac{4 m}{M}-\frac{20}{3} \frac{m}{M} \mathbf{w}^{2}\right) \frac{\mathbf{p}^{2}}{2 m}-4 \frac{m}{M} \frac{w^{i} Q_{P i j} w^{j}}{m}
$$

where $Q_{P i j}=p_{i} p_{j}-\delta_{i j} p^{2} / 3$. Here we have neglected the terms linear and cubic in $\mathbf{p}$ since the experiments in question deal with a bound state, for which such averages vanish.

The corrections in (24) have the same form as those discussed in Ref. 1] and we only focus upon the two terms which are probed by the measurements in clock comparison experiments: 
the velocity-spin coupling and the quadrupolar correction to the inertial mass. The first correction is absent in the D-particle recoil model. In the notation of Ref. [1] this leads to

$$
\Theta_{2}+\frac{1}{2} \Theta_{4}=0
$$

and the model in fact evades the stringent limit settled in that reference. Next we concentrate on the quadrupolar mass correction $\delta h_{Q}=-4 w^{i} Q_{P i j} w^{j} / M$. Comparison with the corresponding term in Eq. (6) of Ref. [1] leads to the identification

$$
\frac{4}{M}=\left|\Theta_{1} \ell_{P}\right|
$$

This differs from the result in Ref. 24], which may be due to our use of standard Observer Lorentz transformations, instead of the modified ones proposed in reference [23]. However, as we have shown in Section II] a Galilean transformation is a particular case of the latter, since it leaves invariant both magnitude and direction of $\mathbf{v}$ to lowest order. Yet the source of our effect is tied to the lack of invariance of $V^{0}$ as shown in Eq. (14).

Moreover, we have included all terms $O(1 / M)$ and $O\left(w^{2}\right)$. Indeed, the sensitivity of modern observational techniques is so high that these terms, which were ignored in Ref. 24], are precisely those which are testable in clock comparison experiments. Our approach, based on standard Lorentz transformations and the corresponding conservation laws, leads to their presence in the non-relativistic Hamiltonian (24).

In fact, it seems very difficult to conceive a scenario in which dispersions relations could break Lorentz invariance while an effective isotropy is preserved, i.e. physics remains independent of the direction of $\mathbf{w}$, in frames moving with respect to the CMB frame. The point is that observer standard Lorentz transformations will map such dispersion relations into direction-dependent terms in the Hamiltonian appropriate for the local laboratory frame.

\section{CONCLUSIONS}

The bound we have previously found for $\Theta_{1}$ in Ref. [1] leads to

$$
M>1.2 \times 10^{5} M_{P}
$$

where $M_{P}$ is the Planck mass. This is, so far, the strongest bound on the mass of the D-particle. Let us note that this value for $M$ is larger by several orders of magnitude than 
the values testable in the gamma ray bursts experiments, given their expected sensitivity. The latest astrophysical limit is $M \sim 6.9 \times 10^{15} \mathrm{GeV} \sim 5.6 \times 10^{-4} M_{P}$ [37], which is $\sim 10^{-8}$ times weaker than Eq. (27).

After averaging over the momenta in the bound state, the effective D-particle recoil velocity is

$$
\mathbf{v} \rightarrow-2 \frac{m}{M} \mathbf{w},
$$

as can be seen from Eq. (13). Using also Eq. (27) we obtain the bound

$$
\frac{|\mathbf{v}|}{c}<2 \times 10^{-27},
$$

for the D-particle recoil velocity after colliding with a $m \approx 1 \mathrm{GeV}$ target moving with the Earth through the cosmic background.

Let us remark that the results of this paper apply to a larger set of quantum gravity models than that of reference 24]. Indeed, let there be a model having a "privileged" vector $U^{\mu}$ such that the Dirac equation takes the form:

$$
\left\{\gamma^{\mu} i \partial_{\mu}-\gamma^{\rho}\left[W_{\rho} U^{\mu}\left(\delta_{\mu}^{\nu}-W_{\mu} W^{\nu}\right)\right] i \partial_{\nu}-m\right\} \Psi=0 .
$$

Then, the reader can verify that, provided that the vector $U^{\mu}$ has the nonrelativistic limit $U^{\mu} \approx(1, \mathbf{u})$, the projected vector $\tilde{V}^{\mu}=U^{\nu}\left(\delta_{\nu}^{\mu}-W^{\mu} W_{\nu}\right)$ with components $\tilde{V}^{\mu}=(\mathbf{w} \cdot$ $\left.\mathbf{u}_{C M B}, \mathbf{u}_{C M B}\right)$ in the non-relativistic limit, has the same properties of $V^{\mu}$ and will satisfy a similar bound to (29) provided $\mathbf{u}_{C M B} \propto \mathbf{p}_{C M B}$.

Our analysis shows that the existing high precision experimental results are able to set very stringent bounds on the mass and the recoil velocity of the D-particle. The latter bound indicates that such velocity is extremely small, even by the standards of every day experience (e.g. the speed of a crawling snail is $\left.\left(\sim 10^{-11} \mathrm{c}\right)\right)$. Although it is in principle conceivable that further refinements could possibly be used to detect the recoil velocity of the D-particle, this last observation suggests that it would be quite unlikely that such effects can ever be detected.

We have found that after identifying the preferred frame of reference associated with Planck scale physics effects with the CMB frame, existing results of atomic and nuclear physics experiments can be translated into very strict bounds on the quantum gravity induced modifications to the propagation of Dirac fields. The present results indicate that using the type of experiments we have considered, string theory inspired schemes are as 
testable as those in loop quantum gravity. Moreover, they seem to cast doubts on the possibility to detect such effects in the near future, using the type of experiments with high energy gamma bursts which have been advocated in [10, 11, 12].

\section{Acknowledgments}

We wish to ackowledge the authors of Ref. [24] for very valuable comments and sev-

eral other colleagues for criticisms. D.S. would like to acknowledge partial support from DGAPA-UNAM Project No IN 112401, a CONACYT sabbatical fellowship, and the Eberly Endowment and thanks Penn State University for its hospitality. L.U acknowledges partial support from DGAPA-UNAM Project No IN-117000 and CONACYT project 40745-F. H. V. acknowledges a leave of absence from Universidad Nacional de La Plata.

[1] D. Sudarsky, L. Urrutia, and H. Vucetich, Phys. Rev. Lett. 89, 231301 (2002), gr-qc/0204027.

[2] V. A. Kostelecky and S. Samuel, Phys. Rev. D39, 683 (1989).

[3] V. A. Kostelecky and S. Samuel, Phys. Rev. D40, 1886 (1989).

[4] V. A. Kostelecky and R. Potting, Nucl. Phys. B359, 545 (1991).

[5] V. A. Kostelecky and R. Potting, Phys. Lett. B381, 89 (1996), hep-th/9605088.

[6] D. Colladay and V. A. Kostelecky, Phys. Rev. D55, 6760 (1997), hep-ph/9703464.

[7] D. Colladay and V. A. Kostelecky, Phys. Rev. D58, 116002 (1998), hep-ph/9809521.

[8] V. A. Kostelecky (2001), hep-ph/0104227.

[9] R. Bluhm (2001), hep-ph/0111323.

[10] G. Amelino-Camelia, J. R. Ellis, N. E. Mavromatos, D. V. Nanopoulos, and S. Sarkar, Nature 393, 763 (1998), astro-ph/9712103.

[11] D. V. Ahluwalia, Nature 398, 199 (1999), gr-qc/9903074.

[12] G. Amelino-Camelia, Lect. Notes Phys. 541, 1 (2000), gr-qc/9910089.

[13] R. Gambini and J. Pullin, Phys. Rev. D59, 124021 (1999), gr-qc/9809038.

[14] J. Alfaro, H. A. Morales-Tecotl, and L. F. Urrutia, Phys. Rev. D66, 124006 (2002), hepth/0208192.

[15] J. Alfaro, H. A. Morales-Tecotl, and L. F. Urrutia, Phys. Rev. Lett. 84, 2318 (2000), gr- 
qc/9909079.

[16] J. Alfaro, H. A. Morales-Tecotl, and L. F. Urrutia, Phys. Rev. D65, 103509 (2002), hepth/0108061.

[17] L. Urrutia, Mod. Phys. Lett. A17, 943 (2002), gr-qc/0205103.

[18] J. R. Ellis, N. E. Mavromatos, and D. V. Nanopoulos, Gen. Rel. Grav. 32, 127 (2000), grqc/9904068.

[19] J. R. Ellis, N. E. Mavromatos, and D. V. Nanopoulos, Phys. Rev. D61, 027503 (2000), grqc/9906029.

[20] J. R. Ellis, K. Farakos, N. E. Mavromatos, V. A. Mitsou, and D. V. Nanopoulos, Astrophys. J. 535, 139 (2000), astro-ph/9907340.

[21] J. R. Ellis, N. E. Mavromatos, and D. V. Nanopoulos, Phys. Rev. D62, 084019 (2000), grqc/0006004.

[22] E. Gravanis and N. E. Mavromatos, JHEP 06, 019 (2002).

[23] J. R. Ellis, N. E. Mavromatos, D. V. Nanopoulos, and G. Volkov, Gen. Rel. Grav. 32, 1777 (2000), gr-qc/9911055.

[24] J. R. Ellis, E. Gravanis, N. E. Mavromatos, and D. V. Nanopoulos (2002), gr-qc/0209108.

[25] C. H. Lineweaver, L. Tenorio, G. F. Smoot, P. Keegstra, A. J. Banday, and P. Lubin, Astrophys. J. 470, 38 (1996).

[26] V. W. Hughes, H. G. Robinson, and V. B. López, Phys. Rev. Lett. 4, 342 (1960).

[27] R. W. P. Drever, Phil. Mag. 6, 683 (1961).

[28] J. D. Prestage, J. J. Bollinger, W. M. Itano, and D. J. Wineland, Phys. Rev. Lett. 54, 2387 (1985).

[29] S. K. Lamoreaux, J. P. Jacobs, B. R. Heckel, F. J. Raab, and E. N. Fortson, Phys. Rev. Lett. $\mathbf{5 7}, 3125$ (1986).

[30] S. K. Lamoreaux, J. P. Jacobs, B. R. Heckel, F. J. Raab, and E. N. Fortson, Phys. Rev. D 39, 1082 (1989).

[31] T. E. Chupp, R. J. Hoare, R. A. Loveman, E. R. Oteiza, J. M. Richardson, M. E. Wangshul, and A. K. Thompson, Phys. Rev. Lett. 63, 1541 (1989).

[32] C. J. Berglund, L. R. Hunter, J. D. Krause, E. O. Prigge, M. S. Ronfeldt, and S. K. Lamoreaux, Phys. Rev. Lett. 75, 1879 (1995).

[33] D. Bear, R. E. Stoner, R. L. Walsworth, V. A. Kostelecky, and C. D. Lane, Phys. Rev. Lett. 
85, 5038 (2000), physics/0007049.

[34] D. F. Phillips et al., Phys. Rev. D63, 111101 (2001), physics/0008230.

[35] V. A. Kostelecky and C. D. Lane, J. Math. Phys. 40, 6245 (1999), hep-ph/9909542.

[36] D. Colladay and P. McDonald, J. Math. Phys. 43, 3554 (2002), hep-ph/0202066.

[37] J. R. Ellis, N. E. Mavromatos, D. V. Nanopoulos, and A. S. Sakharov (2002), astro$\mathrm{ph} / 0210124$. 